\title{
Dynamics of the Magnetic Flux Peculiar to Antiferromagnetic Superconductors
}

\begin{abstract}
T. KRZYSZTOŃ
Institute of Low Temperature and Structure Research Polish Academy of Sciences, 50-950 Wrocław, P.O. Box 1410, Poland

It might be possible that in high temperature layered antiferromagnetic supeconductors an applied magnetic field causes the mixed state to be composed of Josephson vortices possessing magnetic domains. We discuss how this new kind of vortices moves through the intrinsic pinning potential and calculate their activation energy of thermal as well as quantum excitation. We show a possible new phenomenon of crossover from quantum to thermal creep in constant temperature forced by the change of intensity of the external field, or by the change of its direction in the basal plane of layered structure.
\end{abstract}

PACS numbers: 74.60.--w, 74.25.Ha

\section{Introduction}

The layered structure considered in the paper may simulate the real structure of $\mathrm{ErBa}_{2} \mathrm{Cu}_{3} \mathrm{O}_{7}$ and similar antiferromagnetic layered superconductors. The structure consists of superconducting layers of thickness $d_{\mathrm{s}}$ and magnetic moments $M$ of rare earth (RE) ions running parallel and antiparallel to the $b$ axis in the insulating layers of thickness $d_{\mathrm{i}}, d=d_{\mathrm{s}}+d_{\mathrm{i}} \approx d_{\mathrm{i}}$. When an external field is applied parallel to the magnetic moments the antiferromagnetic (AF) phase is stable up to the critical field $H_{T}$ above which spin-flop (SF) phase develops [1]. In the present model we come across two following situations: (1) The external magnetic field is pointing in the $b$ direction (parallel to the RE moments, $x$-axis) and exceeds $H_{T}$ in the vortex core. Then the magnetic subsystem in the core undergoes SF transition; (2) The external magnetic field is applied along the $a$ direction (perpendicular to the RE moments, $y$-axis). Then the AF alignment is unstable against $\mathrm{SF}$ transformation and the vortex state is created in the uniform SF phase. The magnetic structure of a Josephson vortex lying parallel to magnetic moments in the $a b$ plane is shown in Fig. 1. In order to calculate a flux creep rate we use the method of critical nucleus developed in [2]. The activated nucleus consists of a kink-antikink excitation, that is, a vortex line segment of length $R$ is thrown to the adjacent layer, thereby creating a pair of vortex-antivortex pancakes [3]. The 


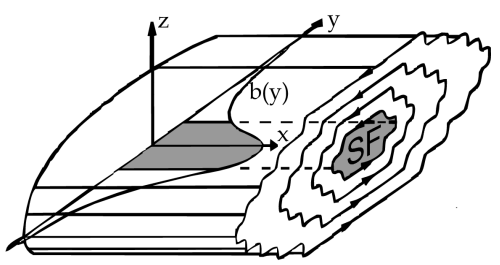

Fig. 1. Magnetic structure of the Josephson vortex.

activation energy $U$ can be regarded as the energy barrier of the intrinsic pinning. Depending on the magnitude of the driving current density $j$ the motion of the flux may continue as single vortex activation or activation of the vortex bundle. When the driving current is weaker than $j_{0}$ (characteristic current due to distortion of the vortex) the critical nucleus is a $3 \mathrm{D}$ object. In our case it is a parallelepiped of the height $R$ along the bundle and of the section $S$ across it.

\section{Thermal activation of the magnetic flux}

The activation energy is a sum of the volume energy due to the Lorentz force and the surface energy:

$$
U_{a, b}=-j B d R S+\delta E_{a, b}\left(\frac{B S}{\varphi_{0}}\right)+j_{0 a, b} d R \sqrt{B S \varphi_{0}} .
$$

The subscript " $a$ " or " $b$ " indicates that the quantity is calculated for $a$ or $b$ direction in the plane. $j_{0 a}=j_{0 b}+5 d\left(\mu_{0} H_{T}+M\right) /\left(128 \lambda_{a b}^{2}\right), j_{0 b}=B d /\left(4 \lambda_{a b}^{2}\right)$, and $B$ is the magnetic induction. The second term is the loss of condensation energy $\delta E$ (and magnetic domain energy in the case of $a$ direction) on both surfaces perpendicular to the bundle multiplied by the number of vortices threading these surfaces. The third term is the elastic energy released in the surface parallel to the shifted vortex $j_{0 a, b} d R \varphi_{0}$ multiplied by the number of shifted vortices $\sqrt{B S / \varphi_{0}}$ (one vortex per plane). The critical nucleus is then $S_{\mathrm{c}}=\left(\varphi_{0} / B\right)\left(j_{0 a, b} / j\right)^{2}, R_{\mathrm{c}}=\delta E_{a, b} /\left(j d \varphi_{0}\right)$, and the activation energy is

$$
U_{a, b}^{\mathrm{c}}=\delta E_{a, b}\left(\frac{j_{0 a, b}}{j}\right)^{2} .
$$

$\delta E_{b}=2 d \epsilon_{0} \ln \left(d / \xi_{\mathrm{c}}\right), \delta E_{a}=(77 / 64) d \epsilon_{0} \ln \left(\varphi_{0} /\left[\pi d^{2}\left(\mu_{0} H_{T}+M\right)\right]\right) \ln \left(d / \xi_{\mathrm{c}}\right)$ and $\epsilon_{0}=\varphi_{0}^{2} /\left(4 \pi \lambda_{a b} \lambda_{c} \mu_{0}\right)$. We consider the motion of activated kinks of the length $L$ in the magnetic field direction along the layers. The mean electric field associated with this motion is given by $E=B P L d S_{\mathrm{c}}$, where $P$ is the activation probability per unit volume and unit time. For thermal activation this probability is $P \sim \exp \left(-U_{\mathrm{c}} / k_{\mathrm{B}} T\right)$. There is however a crossover temperature $T_{0}$ below which the probability of quantum tunneling of vortices is dominating. The preexponential factors and $T_{0}$ cannot be calculated in the framework of thermodynamic considerations alone. Fortunately, it was shown in [4] that the probability of macroscopic quantum excitation is proportional to $j^{3}$. Thus we can assume that 
$P=\alpha_{0} j^{3} \exp \left(-U_{\mathrm{c}} / k_{\mathrm{B}} T\right)$. Now we can calculate the current-voltage characteristics for the current density $j \ll j_{0}$

$$
E_{a, b}=\varphi_{0} d L \alpha_{0} j_{0 a, b}^{2} j \exp \left(-\frac{\delta E_{a, b}}{k_{\mathrm{B}} T}\left(\frac{j_{0 a, b}}{j}\right)^{2}\right) .
$$

This almost linear dependence of $E$ on $j$ indicates that the resistive mechanism of bundle activation follows Ohm law. We can also calculate the rate of flux creep due to the thermal activation of vortices. To do this let us consider hollow cylindrical sample of a radius $r$ and the wall thickness $l \ll r$ placed in the magnetic field $B_{\mathrm{ex}}>B_{\mathrm{c} 1}$ applied parallel to the cylinder axis. The sample has the trapped field $B_{\text {in }}$ inside the hole and corresponding trapped flux $\Phi=\left(B_{\text {in }}-B_{\text {ex }}\right) \pi r^{2}$. According to the Faraday law electric field due to the change of the trapped flux is equal to $\left(\mu_{0} / 2\right) \operatorname{lr}(\mathrm{d} j / \mathrm{d} t)$. Consequently for excitations in the form of bundle of vortices we have the following equation:

$$
\Omega j \exp \left(-\frac{\delta E_{a, b}}{k_{b} T}\left(\frac{j_{0 a, b}}{j}\right)^{2}\right)+\frac{\mathrm{d} j}{\mathrm{~d} t}=0,
$$

where $\Omega=\varphi_{0} \alpha_{0} j_{0}^{2} /\left(\mu_{0} \gamma\right)$ and $\gamma=r l /(L d)$ is the factor determined by the geometry of the sample. The solution of Eq. (4) is given in terms of exponential integrals and for the case of $j_{0 a, b} / j-1 \ll 1$ it can be approximated as:

$$
\frac{\Phi(0)}{\Phi(t)}-1=\frac{k_{\mathrm{B}} T}{2 \delta E_{a, b}}\left(\frac{j(0)}{j_{0 a, b}}\right)^{2} \ln \left(1+\omega_{a, b} t\right)
$$

where $\omega_{a, b}=\frac{4 \varphi_{0} \alpha_{0} \delta E_{a, b}}{\mu_{0} \gamma}\left(\frac{j_{0 a, b}}{j(0)}\right) \exp \left(-\frac{\delta E_{a, b}}{k_{b} T}\left(\frac{j_{0 a, b}}{j(0)}\right)^{2}\right)$. For $0 \ll t \ll 1 / \omega$ the change of trapped flux is linear in time and for $t \gg 1 / \omega$ logarithmic. In antiferromagnetic superconductors, however, we see an additional change of characteristic frequency as the magnetic field changes its direction in the $a b$ plane.

\section{Quantum activation of the magnetic flux}

Below $T_{0}$ the activation probability is $P \sim \exp (-S / \hbar)[5,6]$. In the following we show a considerable change of tunneling rate and crossover temperature due to the SF phase transition in the vortex core. Now, let us consider the vortex line as a straight string-like object of an effective mass per unit length $m$ trapped into a metastable state in an intrinsic pinning potential $V(u)$ and exposed to continuous deformation $u(x, t)$ in the $\hat{z}$ direction. The magnetic field is applied in $\hat{x}$ direction. In the semi-classical approximation the quantum decay rate is calculated as a saddle-point solution (bounce) of the Euclidean action $S$ for the string

$$
\begin{aligned}
S= & \int_{-\infty}^{\infty} \mathrm{d} x \int_{0}^{\hbar \beta} \mathrm{d} \tau\left[\frac{1}{2} m\left(\frac{\partial u}{\partial \tau}\right)^{2}+\frac{\varepsilon_{1}}{2}\left(\frac{\partial u}{\partial x}\right)^{2}+V(u)\right. \\
& \left.+\frac{\eta}{2 \pi} \int_{0}^{\hbar \beta} \mathrm{d} \tau^{\prime}\left|\frac{u(x, \tau)-u\left(x, \tau^{\prime}\right)}{\tau-\tau^{\prime}}\right|^{2}\right]
\end{aligned}
$$


Here $\beta=\left(k_{\mathrm{B}} T\right)^{-1}, \eta$ is the viscosity coefficient and $\tau$ denotes imaginary time. The pinning potential $V(u)$ consists of intrinsic periodic part and the Lorentz potential,

$$
V(u)=-\frac{\varphi_{0} j_{\mathrm{c}} d}{2 \pi} \cos \left(\frac{2 \pi u}{d}\right)-\varphi_{0} j u,
$$

where $j_{\mathrm{c}}$ denotes critical depinning current. The last term in (6) is so-called Caldeira-Leggett action [7] which describes ohmic damping. The line tension $\varepsilon_{1}$ is different for vortices in two different orientations in the $a b$ plane. Those vortices lying parallel to $b$ direction and those ones lying in the $a$ direction but created in the magnetic field less than $\frac{1}{2} H_{T}$ have the line tension $\varepsilon_{b}$ [1], and those ones lying in the $a$ direction but possessing SF domain have the line tension $\varepsilon_{a}[8]$

$$
\varepsilon_{b}=\epsilon_{0} \ln \frac{\lambda_{a b}}{d}, \quad \varepsilon_{a}=\frac{\varphi_{0} H_{T}}{2}+\frac{9}{128} \epsilon_{0} \ln \frac{\varphi_{0} \lambda_{\mathrm{c}}^{2}}{\pi\left(\mu_{0} H_{T}+M\right) d^{2} \lambda_{a b}^{2}} .
$$

In the semiclassical approximation the decay rate is given by the value of the action on a classical trajectory obtained from the Euler-Lagrange equations of the motion. The trajectory $u_{0}(x)$ for static solution gives the activation energy in the thermal regime $T>T_{0}$. Below $T_{0}$ a new kind of trajectory, periodic in imaginary time, develops. Therefore, $u(x, \tau)$ can be expanded in the Fourier series with Matsubara frequencies $u(x, \tau)=u_{0}(x)+\sum_{n=1}^{\infty} u_{n}(x) \cos \left(\omega_{n} \tau\right)$, where $\omega_{n}=2 \pi n / \hbar \beta$. Substituting this expansion into the Euler-Lagrange equations and linearizing the potential around the static solution $u_{0}(x)$ one obtains in the first step of perturbation:

$$
-\varepsilon_{1} \frac{\partial^{2} u_{n}}{\partial x^{2}}+V^{\prime \prime}\left(u_{0}\right) u_{n}=-\left(\eta \omega_{n}-m \omega_{n}^{2}\right) u_{n}
$$

The above equation has three discrete solutions [9], the unstable one corresponding to the tunnelling process determines the crossover temperature

$$
k_{\mathrm{B}} T_{0}=\frac{\hbar \eta}{4 \pi m}\left\{\left[1+\frac{20 \pi \varphi_{0} j_{\mathrm{c}} m}{d \eta^{2}}\left(\frac{j_{\mathrm{c}}-j}{2 j_{\mathrm{c}}}\right)^{\frac{1}{2}}\right]^{\frac{1}{2}}-1\right\} .
$$

\section{Switching between creep regimes at a constant temperature}

The above calculations apply to both kinds of vortices. The only difference is their effective mass and viscosity coefficient. It is possible to express these parameters as a function of condensation energy accumulated in the vortex cores. For the stationary flux flow the viscous force $\eta \partial u / \partial t$ is equal to Lorentz force. The electric field generated by the moving vortex is $E=B \partial u / \partial t$, so we get $E=\left(\varphi_{0} B / \eta\right) j=\rho j=\rho_{\mathrm{N}} B /\left(\mu_{0} H_{\mathrm{c} 2}\right) j$, where $\rho_{\mathrm{N}}$ is the normal phase resistivity in the $a b$ plane and $H_{\mathrm{c} 2}$ is the upper critical field parallel to the layers. Finally, $\eta=\varphi_{0} \mu_{0} H_{\mathrm{c} 2} / \rho_{\mathrm{N}}=\varphi_{0} \kappa H_{\mathrm{c}} \mu_{0} \sqrt{2} / \rho_{\mathrm{N}}=4 \varepsilon_{1} \mu_{0} \sqrt{3} \kappa^{2} /\left(\pi \rho_{\mathrm{N}} \ln \kappa\right)$. The effective mass of the vortex can be deduced from the work of Suhl [10]. He derived the core contribution to the inertial mass $m_{\text {core }}=\frac{3}{8} m_{\mathrm{e}} \xi^{2} H_{\mathrm{c}}^{2} \mu_{0} / \epsilon_{\mathrm{F}}$, where $m_{\mathrm{e}}$ denotes the mass of the electron and $\epsilon_{\mathrm{F}}$ is the Fermi energy, and the electromagnetic contribution 
coming from the energy of the electric field induced by the moving flux. Simple estimation shows that this contribution in layered superconductors is $10^{-4}$ of the core contribution. Therefore $m=9 \varepsilon_{1}^{2} \lambda_{a b}^{2} m_{\mathrm{e}} \mu_{0} /\left[\varphi_{0}^{2} \pi^{2} \epsilon_{\mathrm{F}}(\ln \kappa)^{2}\right]$. It is possible now to relate the crossover temperature in Eq. (10) to the line tension of the vortex $T_{0}=\alpha \varepsilon_{1}^{-1}$. The coefficient $\alpha$ depends on the material constants and critical current intensity which is assumed equal in both directions. As was mentioned there are two types of vortices in the system. The first ones, without magnetic domain, occur when the field is applied in the $a$ direction, but its intensity does not exceed $\frac{1}{2} H_{T}$ or in the $b$ direction. For those vortices crossover temperature is $T_{0 b} \sim \varepsilon_{b}^{-1}$. The other vortices, possessing magnetic domain, occur when the field is applied in the $a$ direction and its intensity exceeds $\frac{1}{2} H_{T}$. For those vortices the crossover temperature is $T_{0 a} \sim \varepsilon_{a}^{-1}$. It is easy to see that $\varepsilon_{a}>\varepsilon_{b}$ and therefore $T_{0 b}>T_{0 a}$. Hence it is possible to switch the creep regime at a constant temperature. To do this, one needs to change the field intensity or simply change the field direction in the $a b$ plane. The diagrams in Fig. 2 show possible scenarios of crossover from

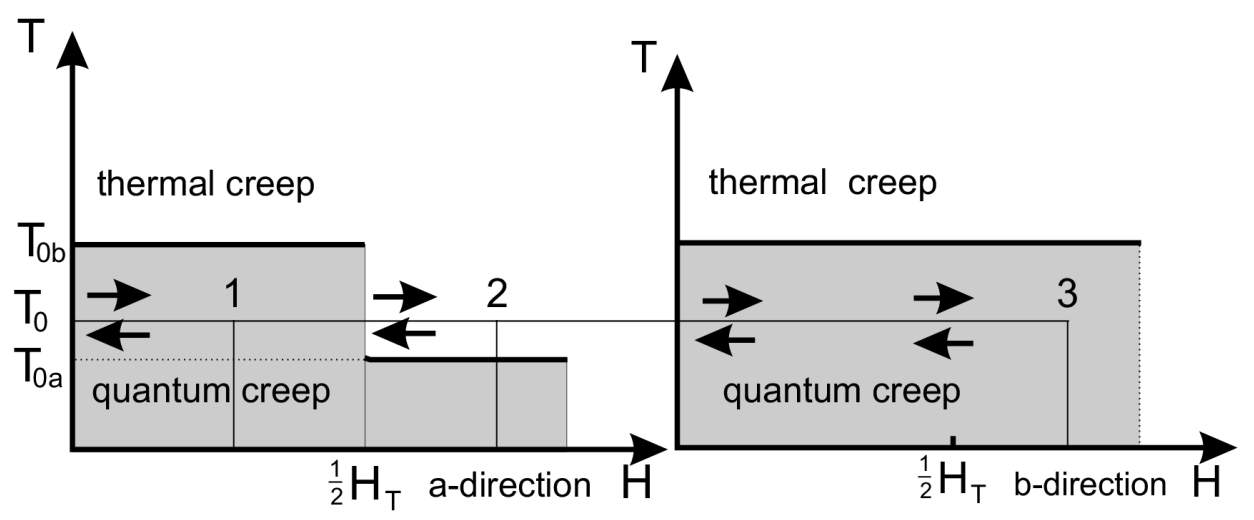

Fig. 2. Possibility of switching between creep regimes at a constant temperature.

quantum to thermal regimes. Let us discuss just two of them. The first prescription is the following. Let us fix the temperature $T_{0}$ somewhere in the range $T_{0 b a}>T_{0}>T_{0 a}$. Then align the external field in the $a$ direction and increase its intensity to the point marked " 1 " on the left diagram in Fig. 2. The system is in the quantum creep regime now. Then increase the external field beyond $\frac{1}{2} H_{T}$. The system jumps to the point " 2 " of the left diagram and finds itself in the thermal creep regime. Doing the same operations in the reverse order one enforces the system to crossover from thermal to quantum creep regime. The other scenario is the following. Let us apply magnetic field along $a$ axis and increase its intensity above $\frac{1}{2} H_{T}$ keeping temperature constant in the interval $T_{0 b}>T_{0}>T_{0 a}$. Then move the direction of the external field from $a$ to $b$ axis. The system goes now from the point "2" of the left diagram (thermal creep) to the point " 3 " of the right diagram (quantum creep). 


\section{Acknowledgments}

This research is supported by the State Committee for Scientific Research (KBN) within the Project No. 3 T10A 00126.

\section{References}

[1] T. Krzysztoń, Phys. Lett. A 190, 196 (1994).

[2] S. Chakravarty, B.I. Ivlev, Y.N. Ovchinnikov, Phys. Rev. B 42, 2143 (1990).

[3] T. Krzysztoń, Physica C 294, 47 (1998).

[4] P. Tekiel, Z. Phys. B 104, 423 (1997).

[5] B.I. Ivlev, Yu.N. Ovchinnikov, R.S. Thompson, Phys. Rev. B 44, 7023 (1991).

[6] W.M. Gaber, B.N. Achar, Phys. Rev. B 52, 1314 (1995).

[7] A.O. Caldeira, A.J. Leggett, Ann. Phys. (N.Y) 149, 374 (1983).

[8] T. Krzysztoń, Physica C 340, 156 (2000).

[9] L.D. Landau, E.M. Lifshitz, Quantum Mechanics, Pergamon Press, Oxford 1962.

[10] H. Suhl, Phys. Rev. Lett. 14, 226 (1965). 